Wang Z., He B., Yang Y., Shen C. and Peña-Mora F. (2020). "Building a next generation AI platform for AEC: a review and research challenges" In: Proc. 37th CIB W78 Information Technology for Construction Conference (CIB W78), São Paulo, Brazil, pp. 27-45. DOI: http://dx.doi.org/10.46421/27066568.37.2020.paper003

\title{
BUILDING A NEXT GENERATION AI PLATFORM FOR AEC: A REVIEW AND RESEARCH CHALLENGES
}

\author{
Zihao Wang ${ }^{1 *}$, Bingzhen $\mathrm{He}^{2 *}$, Yu Yang ${ }^{3}$, Charles Shen ${ }^{4}$, and Feniosky Peña-Mora ${ }^{5}$
}

\begin{abstract}
Transformation of the AEC industry has been found to face significant and persistent hurdles both internal and external to the industry, such as industrial fragmentation, difficulty in talent recruiting, inadequate collaboration and knowledge transfer. Meanwhile, advances and maturity in digital technologies recent years offer tremendous potential to tackle these challenges. Examples include Artificial Intelligence (AI) and deep learning, computer vision, big data analytics, Building Information Models (BIM), 3D scanning, Augmented Reality (AR), Unmanned Aerial Vehicles (UAVs), autonomous driving, and blockchain.

We envision that the next generation platform for the AEC industry shall integrate this diverse list of digital technologies. In this paper, we review the stateof-the-art in related literature aiming at creating such an integrated platform. Our review is organized into the following four themes, and for each of them, we also identify gaps and challenges.

1. AI-based BIM model generation: this topic addresses how to make BIM models more ubiquitous, which includes the automatic generation of As-planned BIM model from 2D drawings and the automatic generation of As-built BIM model from site images/videos and point clouds.

2. Blockchain-based collaboration: this theme explores the possibilities of applying Blockchain for AEC to improve various essential aspects such as trust in multi-party collaboration and contract enforcement.

3. AR-based visualization: this topic studies the AR-based visualization for construction management, which aims to increase the efficiency of information transmission.

4. AI-based Construction management: this area reviews the application of AI in progress monitoring, safety management, quality management, and contract management.

Based on the above analysis, we conclude by outlining potential topics of a research framework for creating a next generation AI platform for AEC.
\end{abstract}

Keywords: Artificial Intelligence, BIM, Blockchain, Augmented Reality, Construction Management.

1 M.S. Candidate, Department of Civil Engineering and Engineering Mechanics, Columbia University, New York, USA, zw2588@columbia.edu

2 M.S. Candidate, Department of Civil Engineering and Engineering Mechanics, Columbia University, New York, USA, bh2724@columbia.edu

3 M.S. Candidate, Department of Civil Engineering and Engineering Mechanics, Columbia University, New York, USA, yy2973@columbia.edu

4 Research Scientist, Advanced Construction and Information Technology Laboratory, Columbia University, New York, USA, charles.shen@columbia.edu

5 Edwin Howard Armstrong Professor, Department of Civil Engineering and Engineering Mechanics, Columbia University, New York, USA, feniosky@columbia.edu

* Zihao Wang and Bingzhen He contributed equally to this work 


\section{INTRODUCTION}

The Architecture, Engineering, and Construction (AEC) industry is one of the largest industries in the world; yet it is also known to have the lowest productivity gains of any industry in the past decades. World Economic Forum (WEF, 2016) reports estimate that a $1 \%$ rise in the construction industry productivity worldwide could save $\$ 100$ billion a year.

Nowadays traditional construction management is facing a series of challenges. It is expensive and time consuming for managers to monitor project performance (Pučko et al. 2018). It is difficult for managers to monitor projects in real time (Navon and Sacks 2007). Another problem is the trust and transparency crisis between the parties in the project (Hargaden et al. 2019). Meanwhile, due to the lack of visualization technology, site information cannot be transmitted to managers effectively, which may lead to potential mistakes in decision making (Sun et al. 2020).

In the past few years, researchers have tried to address these challenges by introducing digital technology into the AEC industry, including Artificial Intelligence (AI), Computer Vision, Building Information Modeling (BIM), Blockchain, Augmented Reality (AR), etc. Artificial Intelligence (AI) is defined as the intelligence of machines, i.e. replicate human intelligence via artificial technologies to invent intelligent machines (Minsky 1960; Mohasses 2019). In the AEC industry, AI technology becomes popular in resolving problems or improve performance in construction projects, e.g. machine learning, which is increasingly employed in project delay risk prediction (Gondia et al. 2019), facility life-cycle cost analysis (Gao et al. 2019), etc. Deep learning is one subdomain of machine learning which applies deep neural networks in task learning. Integrated with computer vision, deep learning has been used in safety management (Fang et al. 2020), progress monitoring (Lei et al. 2019), and 3D model reconstruction (Che et al. 2019). Building Information Modelling (BIM) is an information management system of buildings during their full lifecycle (Delgado et al. 2018). The application of BIM promotes the booming of the AEC industry, as it helps improve the efficiency and reduce costs by developing virtual 3D models and effective management systems with rich information. Blockchain, which originates from Bitcoin (Nakamoto 2008), is a distributed public ledger that the data, assets and transaction records can be shared among participants in the network to achieve mutual-trust networks (Hargaden et al. 2019). The potentials of applying blockchain have been explored across some construction domains, e.g. construction supply chain (Tezel et al. 2019). Augmented Reality (AR) is the interaction between the artificial elements generated from digital devices and the real-life environment (Mitha et al. 2013). Now, AR has already been used in progress monitoring (Ratajczak et al. 2019) and defects detection (Lee et al. 2012). However, existing technologies are still fragmented and require integration to perform efficiently in construction management.

This paper provides a literature review and organized these advanced technologies into a next generation AI platform for the AEC industry. The current states of various management to be achieved by the platform are reviewed as well as the possibility of applying AI technology. Meanwhile, existing challenges and future works are discussed. Section 1 gives an overview of related technologies; Section 2 presents the details of the research method; Section 3 shows a review of the technologies that contribute to the next generation AI platform and current states of construction management; Section 4 concludes the work in this paper. 


\section{Methodology}

The major databases used by this paper include Web of Science, ScienceDirect, ASCE, IEEE, Google Scholar. In the literature search, we used different sets of keywords for different topics to obtain a list of related publications (See Table 1). By limiting the publication data from 2014 to the present, the publications that represent the latest research findings are collected. This review focuses on journal articles and conference papers in artificial intelligence, computer vision, civil engineering, and construction management. To select highly related publications, the collected publications are then filtered through reading abstracts and conclusions to exclude the papers that are not related to construction management. After the two-step searching and filtering, a total of 225 academic papers are selected. Due to space constraints, this paper is unable to present a complete review. Therefore, we select a part of these papers (84 papers) to review.

Table 1: Overview of Literature Search

\begin{tabular}{ccc}
\hline Topic & Keyword & No. of Papers \\
\hline $\begin{array}{c}\text { As-planned BIM Model } \\
\text { Generation } \\
\text { As-built BIM Model } \\
\text { Generation }\end{array}$ & $\begin{array}{c}\text { Point clouds, construction, BIM, machine learning, } \\
\text { deep learning, registration, segmentation, } \\
\text { classification, 3D reconstruction }\end{array}$ & 90 \\
$\begin{array}{c}\text { Blockchain-based } \\
\text { Collaboration }\end{array}$ & $\begin{array}{c}\text { Blockchain, AI, IoT, smart contract, BIM, } \\
\text { construction }\end{array}$ & 20 \\
$\begin{array}{c}\text { AR-based Visualization } \\
\text { AI-based Construction } \\
\text { Management }\end{array}$ & $\begin{array}{c}\text { Augmented reality, AI, construction management } \\
\text { Machine learning, deep learning, progress }\end{array}$ & 21 \\
\hline
\end{tabular}

\section{NeXt GENERATION AI PlatForm FOR AEC INDUSTRY}

In this paper, we reviewed the advanced technologies relating to the next generation of AI platforms for the AEC industry. This paper will follow the structure of Figure 1 to review.

- In section 3.1, this paper reviews the methods of input data acquisition.

- In section 3.2, the generation of BIM model is reviewed, including As-planned BIM model generation (Section 3.2.1) and As-built BIM model generation (Section 3.2.2).

- In section 3.3, blockchain-based collaboration is reviewed.

- In section 3.4, this paper reviews AR-based visualization for construction management.

- In section 3.5, this paper reviews AI-based construction management, including progress management (Section 3.5.1), safety management (Section 3.5.2), quality management (Section 3.5.3) and contract management (Section 3.5.4). 


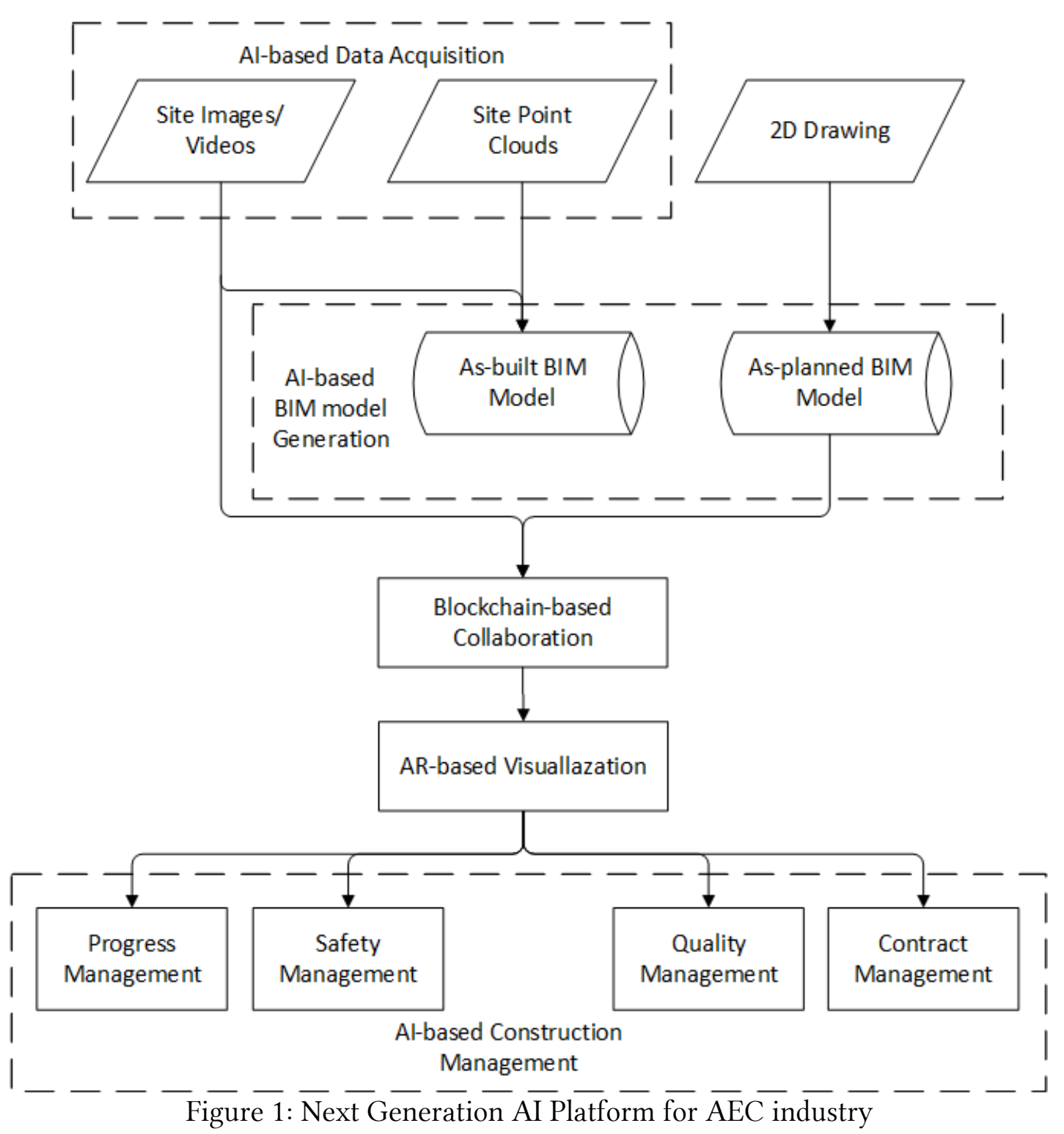

\subsection{AI-based Data Acquisition}

Most of the papers reviewed in this paper require site images/video or point clouds as input. Manual-based data acquisition methods are time-consuming and costly. Unmanned aerial vehicles (UAVs) as a new technology can collect image data in the inaccessible areas or undertake tasks that are dangerous to human beings (Shang and Shen 2017), which is more efficient and cost-effective. More importantly, the images collected via UAVs can provide sufficient visual coverage overlaps of the site to support the generation of 3D building models, which is critical for automated progress monitoring. However, it is still a challenge to achieve autonomous path planning and navigation for UAVs (Ham et al. 2016). Some studies have explored the potentials of applying AI in autonomous path planning (Aggarwal et al. 2019; Hamledari et al. 2018). For instance, according to Hamledari et al. (2018), swarm intelligence was employed in making the UAVs inspection plan, which reduces the flight duration and thus improves the efficiency of site inspection. Besides, some studies integrated 4D BIM with UAVsenabled 3D reconstruction to achieve autonomous path planning of UAVs (Shang and Shen 2017). Using 4D BIM as a prior model, together with ray-tracing to detect visible 
elements, the optimal flight missions can be created at locations where have expected changes to optimize the visual coverage of the flight plan (Ibrahim and Golparvar-Fard 2019). However, the automatic integration of as-built conditions into $4 \mathrm{D}$ BIM is still a primary challenge for UAV-based inspection (Hamledari et al. 2017). To resolve this challenge, Hamledari et al. (2017) utilized industry foundation classes (IFC) schema to achieve automatic integration of UAV-based as-built inspection results into 4D BIM.

\subsection{AI-based BIM Model Generation}

As-planned BIM model shows the proposed progress and As-built BIM model shows the actual progress (Son et al. 2017). These two models are used as inputs in progress management (Golparvar-Fard et al. 2010). In this section, the automatic generation of As-planned BIM models and As-built BIM models will be reviewed. We also discussed the limitations of current technologies.

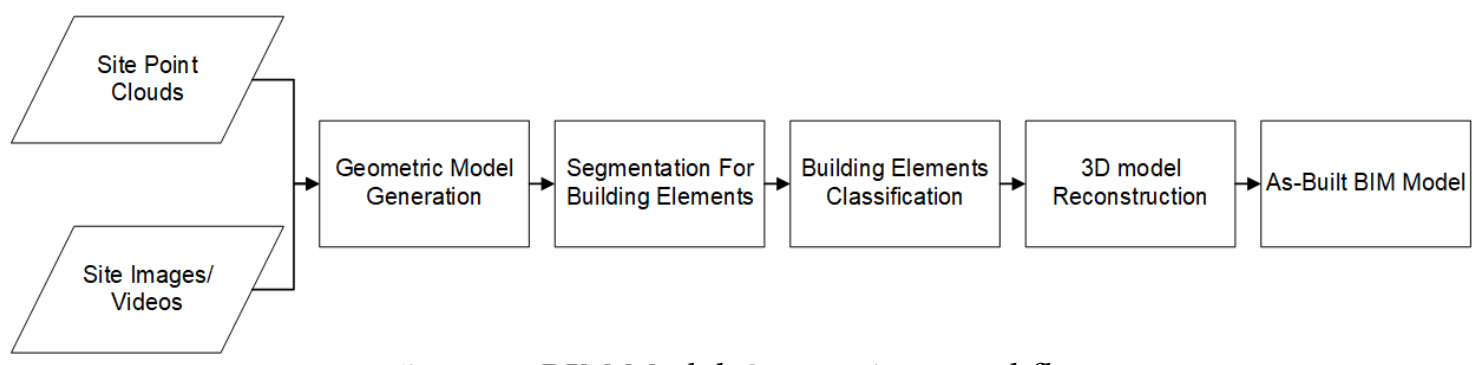

Figure 2: BIM Model Generation Workflow

\subsubsection{As-planned BIM Model Generation}

BIM models can bring tremendous benefits to the AEC industry, but traditional BIM models rely on manual generation (Seyis 2019). To solve this problem, the idea of automatically generating BIM models was proposed. Nowadays there are several methods to generate As-planned BIM model through 2D drawings (Cho and Liu 2017; Lim et al. 2018). However, some challenges exist, e.g. low accuracy of automatic recognition, heavy manual workload (Liu et al. 2017).

Deep learning is used for further automation. A method based on deep learning and integer programming to restore geometric and semantic information of floor plans was proposed (Liu et al. 2017). This method achieved 90\% accuracy, higher than other methods in the same period. However, this algorithm lacks robustness for unseen elements. A deep multi-task neural network was proposed to identify room types and walls (Zeng et al. 2019). Compared with the Raster-to-Vector algorithm (Liu et al. 2017), this algorithm has higher precision. And it can handle more complex floor plans, including irregular shaped rooms and curved walls. The identification results can be further used for 3D reconstruction. However, this method lacks accuracy for inside and outside region identification and requires a larger dataset for training to deal with the diversity of floor plans.

At present, deep learning-based BIM model generation from 2D drawings is still facing some problems. Firstly, the training database is relatively small compared with training databases in other fields of computer vision. Meanwhile, the establishment of databases still rely on manual work. Secondly, the accuracy and generality of the current algorithm needs to be improved. Thirdly, most of the algorithm focus on $2 \mathrm{D}$ drawing recognition and vectorization. Finally, BIM models generated from 2D drawings lack schedules. 


\subsubsection{As-built BIM Model Generation}

The procedure of converting vision-based data (e.g. images, videos) to the as-built BIM model is referred to as Scan-to-BIM. The typical workflow involves four steps: 1) Geometric model generation; 2) Segmentation for building elements; 3) Building elements classification; 4) 3D model reconstruction. Firstly, by registering multiple laserscanning point clouds or generate point clouds from a series of images, a geometric model is built. In the second step, the registered point clouds are segmented into many clusters that have similar properties. In the third step, each cluster is assigned to a class label based on geometric and contextual features observed. With geometric and contextual features, the clusters can be converted to various 3D objects, e.g. wall, windows, floor. The fourth step involves the splice of 3D objects to a realistic BIM model based on different spatial relationships. Then, some other non-metric attributes like types of materials are added to the individual BIM elements. A 3D BIM model is reconstructed successfully. Accurate as-built models can help achieve automatic progress monitoring and contract execution.

\section{(1) Geometric Model Generation}

The input of geometric reconstruction can be classified into point clouds and images. Laser scanning can generate a dense and accurate point cloud, but it also needs professional technicians and expensive equipment (Asadi et al. 2019). For images as inputs, Structure-from-Motion (SfM) and Multi-View Stereo (MVS) are used for reconstruction, which can be got from cheaper digital cameras, become possible (Han et al. 2018).

Deep-learning-based approaches were introduced to try to replace traditional methods in point cloud registration. Based on PointNET (Qi et al. 2017) algorithm, PointNetLK combined the LK algorithm (Lucas and Kanade 1981) which was used for image alignment to register (Aoki et al. 2019). PoinNetLK is more efficient in computing large-scale point sets and it shows good applicability when facing the untrained shape. Deep Closest Point (DCP) algorithm (Wang and Solomon 2019) used a learning-based method to solve the problems existing in the ICP (Besl and McKay 1992) algorithm. When using the ModelNet40 database to test, the DCP algorithm is robust when facing the noise, and its accuracy is higher than PointNetLK (Wang and Solomon 2019). Another method used 3D-CNN and 2D-CNN for coarse registration and fine registration respectively (Chang and Pham 2019). For accuracy, this method is close to RANSAC + ICP algorithm, and its efficiency is 15 times that of RANSAC + ICP algorithm.

In image-based 3D reconstruction, deep-learning-based SfM algorithms are used to solve depth and motion. DeMoN is a convolutional network with an iterative structure (Ummenhofer et al. 2017). However, DeMoN can only handle one image pair, which limits its use. DeepTAM extended the input to multiple images and achieved better performance than DeMoN (Zhou et al. 2018). Other algorithms include DeepSFM (Wei et al. 2019), ENG (Dharmasiri et al. 2018), etc. The accuracy of all these methods is higher than the SIFT-based method.

For MVS, deep-learning-based MVS includes, DeepMVS (Huang et al. 2018), MVSNet (Yao et al. 2018), R-MVSNet (Yao et al. 2019), etc. DeepMVS performed better than the non-learning algorithm COLMAP (Schönberger et al. 2016) in the test. However, it only generates depth maps and still needs post-processing. R-MVSNet (Yao et al. 2019) tried to solve the scalability problem in MVSNet (Yao et al. 2018) by using gated recurrent units to regularizes the cost volume. R-MVSNet (Yao et al. 2019) achieves better reconstruction completeness and overall quality. However, the depth maps fusion 
algorithms in MVSNet (Yao et al. 2018) and R-MVSNet (Yao et al. 2019) are not using deep learning.

(2) Segmentation for Building Elements

After obtaining the complete point clouds of the building from site images/videos or point clouds fragments, the building elements need to be generated. In segmentation, point clouds are segmented into separate point clusters with similar properties based on non-semantic information (e.g. location, geometry, intensity). Some popular machine learning approaches for segmentation include K-nearest neighbors (KNN) and Principal Component Analysis (PCA). The segmentation process laid a foundation for the processing and analysis of each segment or object with richer information, e.g. geometric features and contextual features of objects. However, there is no deep learning-based approach for segmentation, which requires further research on it.

(3) Building Elements Classification

After segmentation, the building elements can be generated and identified. In classification, each cluster is assigned to a class ("label") based on different criteria. Some features need to be extracted to describe point clouds, e.g. geometric and contextual features (Che et al. 2019). Geometric features describe local attributes of building elements, e.g. shape, size. Contextual features involve the various relationships among building elements (e.g. proximity), which makes the classification more robust (Bassier et al. 2019; Che et al. 2019). Since AI technology becomes increasingly popular in recent years, machine learning (ML) is employed in the classification of point clouds as a classifier. Support Vector Machines (SVM), Decision Trees, Random Forest (RF), and Neural Networks (NN) are popular models in classification.

SVM performs well in classifying indoor and outdoor point clouds. In the study of Perez-Perez et al. (2019), SVM was used to extract the relationship between semantic and geometric labels for the following classification of segments into semantic categories. Their method pioneeringly identifies beams and columns automatically in a Scan-to-BIM process with average fitting error of $0.633 \mathrm{~mm}$. However, this approach is highly dependent on the performance of the segmentation method selected. Random forest (RF) consists of multiple decision trees and allows them to select the most popular class (Breiman 2001). In the study of Park and Guldmann (2019), the RF algorithm was employed to classify LiDAR points into four different categories of building elements, reaching a high accuracy (96.5\%). RF classifier is more robust to the quality of training examples and overfitting than other ML classifiers (Belgiu and Drăguţ 2016). However, the sample imbalance among different object classes can affect RF performance, which causes the issues of over- or under-representation of certain classes (Belgiu and Drăguţ 2016).

Deep learning has been considered for the classification of building elements due to its good performance (Che et al. 2019; Chen et al. 2019a; He et al. 2016; Czerniawski and Leite 2019). Although deep learning bypasses manual feature descriptors (Chen et al. 2019a), regular and structured data are required as input, e.g. 2D images. Therefore, there are two main categories: 1) 2D image-based classification (Lawin et al. 2017); 2) 3D voxelization (Maturana and Scherer 2015). In 1), the point clouds are projected or rasterized to $2 \mathrm{D}$ images, while the data are voxelized to $3 \mathrm{D}$ grids in 2). Both two directions mainly use Convolutional Neural Networks (CNNs) as architecture. Lawin et al. (2017) used 2D images to achieve classification of 3D point clouds. They firstly projected 3D point clouds onto synthetic images and then projected the prediction scores obtained from 2D CNN (fed synthetic 2D images) back to the point clouds to achieve the 
classification. Maturana and Scherer (2015) integrated 3D CNN with occupancy grid (named "VexNet") to identify objects like chairs. However, for 3D voxelization, occlusions and clutters impede the object-level segmentation. Besides, it is more complex to use voxel grids as input format, which leads to the loss of accuracy. Moreover, point clouds can be directly used as input but cannot clearly define object boundary except using bounding boxes (Chen et al. 2019a; Qi et al. 2017). Therefore, this method performs well only under the condition that objects are separated from the background, e.g. outdoors.

Both machine learning and deep learning need large datasets of labeled objects for training. Currently, the building elements are labeled by manual work, which is laborintensive and time-consuming (Perez-Perez et al. 2019; Wang et al. 2015). 4D BIM is one possible solution. BIM as a semantically rich model is used in semantic labeling and classification by transferring semantics from BIMs to point clouds. In the study of Braun and Borrmann (2019), as the as-planned BIM model is aligned with the generated point clouds, each BIM element could be projected onto the point clouds. This ensures a precise process of establishing a dataset of labeled building elements. Czerniawski and Leite (2019) extracted the geometry and attached semantics from BIMs and then attached them to the laser scanning data to create a large labeled dataset for training deep neural networks (DNN), which avoids the step of the dedicated annotation.

(4) 3D Model Reconstruction

Once the point clouds have been classified into different categories of building elements, the 3D model reconstruction starts. All the identified building elements are connected and assembled to 3D BIM models. This process needs to consider different spatial relationships, including aggregation (i.e. part of, belong to, etc.), topological (i.e. connected to, inside, outside, etc.), and directional (i.e. above, below, etc.) relationship (Bassier et al. 2019; Hichri et al. 2013). For example, Nguyen et al. (2005) showed an automatic way to deduce topological relations among building elements. Besides, other studies also represented identified objects in IFC format to establish topological relations (Macher et al. 2017). Moreover, Tran et al. (2018) presented a shape grammar approach to generate indoor 3D models with rich topological relations. Apart from spatial relationships, some non-metric attributes are added to individual building elements. These attributes enrich the description of the elements, including the information of materials, historical records, economic data, etc (Hichri et al. 2013). Although no deep learning-based approach is applied in the final reconstruction, some processes can potentially employ deep learning algorithms, e.g. the process of splicing based on spatial relationships.

In the BIM model generation phase, knowledge is still fragmented. At present, it lacks an end-to-end algorithm to directly realize the generation of BIM models. Noise and occlusions are a major challenge for Scan-to-BIM. The current state-of-the-art algorithms show robustness to Gaussian noise, but their performance still needs to be improved when facing a higher level of noise. Occlusions are inevitable in data collection. At present, there is still no effective method to recover the actual state of the object from occlusions. Another challenge is the scalability of the algorithm. The employment of 3D $\mathrm{CNN}$ leads to an increase of cost volume. Many algorithms are limited by the size of memory, so they cannot be applied to large scale reconstruction. The generalization capability of the algorithm also needs to be addressed. AI-based algorithms need to be trained by using existing datasets, which are often standardized. But the diversity of architectural design makes it difficult to set up a dataset which concludes all kinds of 
design and elements. Therefore, the algorithm needs to have a strong generalization ability to face the non-standardized building.

\subsection{Blockchain-based Collaboration}

Blockchain has the potentials to resolve the problems in construction projects, e.g. poor regulation compliance, inadequate collaboration, and complex payment practices ( $\mathrm{Li}$ et al. 2019a). With the integration of BIM and IoT, blockchain can achieve a smart and transparent collaboration platform with advanced functions for construction management, e.g. automated progress monitoring (AI technology) and contract execution (smart contract) (Li et al. 2019a), which significantly reduces the management cost and improves the efficiency of processing.

Some researchers have explored the potentials of integrating blockchain with AI in the AEC industry. Tang et al. (2019) proposed a framework with the integration of blockchain, BIM, IoT, AI, big data. In this framework, based on the data collected by BIM model and IoT sensors, AI algorithms can be used in automated decision making to achieve smart project monitoring. Blockchain can record the decisions and progress updates and ensure the immutability and traceability of data (Singh and Ashuri 2019). Moreover, AI was also considered integrated with blockchain and smart contracts in automated code compliance checking processes (Nawari and Ravindran 2019b). AI can extract related information from regulations into rules automatically, while blockchain guarantees the reliability of the extracted information and the activities of transferring. However, few frameworks have been designed and implemented in real projects (Singh and Ashuri 2019).

Blockchain is in the early stage of development and thus has numerous future works to do. Despite the benefits it would bring to the AEC industry, blockchain is still facing some challenges. Firstly, it is difficult for blockchain and AI to be adopted rapidly by the traditional industry, since the existing mechanism has run well for decades or even hundreds of years. Secondly, there is no actual design for the integration of blockchain and AI technology currently, and the use case for it is even further away. Thirdly, the costs of transferring to the new style from the traditional mechanism are unpredictable and whether it is worth remains to be seen. Furthermore, most research focus on the financial or transaction part of the construction projects, while few researches pay attention to the design and construction part. This part needs high reliability and traceability of the information delivered to guarantee the key criteria of projects (time, cost, quality) and the liability distribution of involved parties. Therefore, there are still lots of work needed to do in the future.

\subsection{AR-based Visualization}

The development of augmented reality (AR) technology provides tools for construction visualization. Golparvar-fard et al. (2010) superimposed the BIM model with the site photos, and showed the difference between the construction progress and the planned progress by using color code. Zollmann (2014) developed an AR system that supports construction site progress monitoring and recording, but it relies on specific hardware. In AR4C system, exclude progress, KPI information is also showed (Ratajczak et al. 2019).

AR technology is also used for defect detection (Lee et al. 2012). ARCam was used for the inspection of steel columns but still had accuracy problems. Park et al. (2013) mapped the BIM model to the site through a marker-based AR system to help the workers confirm the task, and give feedback to the manager. Lamsal and Kunichika 
(2019) used AR marker points and structure sensor SDK to register AR images. This system is used to help workers check their work through the iPad to prevent mistakes.

In AR, deep learning was used for localization. Through using $\mathrm{CNN}$, real-time images can be compared with BIM rendered images to achieve indoor localization (Ha et al. 2018). An AR system based on this method was used for facility management (Baek et al. 2019). Localization can also be achieved by calculating camera pose.

Fast and accurate localization is a problem for AR technology. Due to limited performance, AI-based methods are difficult to be applied to mobile devices (Li et al. 2019b). The current AR system can only display limited information. In the assumption of this paper, AR can be used to display more construction information, such as material list, schedule, construction drawings, etc. Meanwhile, the current system lacks interactivity. When managers click on architectural elements on the screen, more details should be provided.

\subsection{AI-based Construction Management}

\subsubsection{Progress Management}

Automated progress monitoring is achieved by using AI to compare as-built and asplanned BIM model. Comparison methods include geometry-based methods and imagebased methods, which can also be used in combination (Han et al. 2018).

For geometry-based progress monitoring, the first step is to align the models. This step can be done by aligning two point clouds, which is similar to the point cloud registration process. In section 3.2.2 (1), we have discussed the point clouds registration algorithm based on deep learning and explained its advantages. After the alignment, by performing different operations on the two models, progress can be identified. In this process, an identification threshold needs to be set for each building element, so that when the occupancy of the as-built model reaches a certain level, the element is recognized as completed (Golparvar-Fard et al. 2010). This process can be abstracted into a classification process in which AI technology can be used. SVM has been already used as a classifier in this process and has $82.89 \%$ detection accuracy (Golparvar-Fard et al. 2010).

In addition to geometric methods, progress monitoring based on appearance can also be used. When the corresponding materials are identified in both models, the construction progress is detected (Han and Golparvar-Fard 2014). An image-based progress detection algorithm was proposed (Han and Golparvar-Fard 2014), which detects the progress state through the recognition result of the SVM classifier. Another paper also used this method for progress monitoring (Han and Golparvar-Fard 2015). The result showed that the accuracy reached 95.6\% (Han and Golparvar-Fard 2015).

For the application of AI in progress management, some challenges exist. Existing progress monitoring techniques rely on the compartment between as-planned models and as-built models, so it faces similar challenges like occlusions, noise, etc. Progress monitoring is not accurate when as-planned models lack details (such as lack of formwork, shoring, etc.). Meanwhile, the existing monitoring technology only used the low-level knowledge, construction sequencing and other high-level reasoning is not used.

\subsubsection{Safety Management}

Safety is always a significant topic in the AEC industry (Nath et al. 2020). Current research focus on various fields (Fang et al. 2020): failure of wearing Personal protective equipment (PPE) (Fang et al. 2018; Wu et al. 2019), unsafe behavior (Ding et al. 2018; 
Fang et al. 2019), and exposure to hazardous area (Roberts et al. 2017; Zhang et al. 2019). Integrating with computer vision and AI technology, vision-based approaches have become more popular (Nath et al. 2020). It uses cameras to record site images or videos, and then analyzes them by to detect unsafe conditions.

(1) Failure of wearing PPE

Failure of wearing PPE has become one of the main reasons for injuries accidents on construction sites (Li et al. 2017). One approach uses handcrafted features extracted from images or videos to automatically detect the PPEs wearing, e.g. Histogram of Oriented Gradients (HOG) (Dalal and Triggs 2005). Fang et al. (2018) employed Faster R-CNN to automatically detect the non-hardhat-use (NHU) of construction workers. Despite the low precision for identifying the hardhat under the impact of occlusions, the recognition precision and recall rates were consistently over $90 \%$. However, the heavy dependence on the information from upper features might do harm to the performance at different scales in the images. To resolve these problems, Wu et al. (2019) employed a single-stage approach based on the SSD framework to detect hardhats worn by workers, which uses a single $\mathrm{CNN}$ to create bounding boxes.

\section{(2) Unsafe Behavior}

Unsafe behavior of workers leads to around $88 \%$ of the accidents on site (Ding et al. 2018). Ding et al. (2018) integrated Long Short-Term Memory (LSTM) model (Hochreiter and Schmidhuber 1997) with CNNs to identify the potential unsafe behaviors of workers such as climbing a ladder. The accuracy of detecting safe and unsafe behaviors was $97 \%$ and $92 \%$ respectively, which exceeds the performance of other common methods like HOG by about $10 \%$ on average. In addition, R-CNN was also employed in Fang et al. (2019) to detect workers traversing structural supports during construction. As a result, the precision and recall rates reached $75 \%$ and $90 \%$ respectively. However, occlusions and overfitting still influence the results figured out by the model.

(3) Exposure to Hazardous Area

Exposure to hazardous area is another reason for construction accidents. Some sensorbased approaches are used, integrating with AI technology. Zhang et al. (2019) used smartphones to detect near-miss falls by analysing the acquired data with ANN. As a result, the average precision and recall rates were around $90 \%$ and $91 \%$ respectively. Concerning vision-based approaches, in Roberts et al. (2017), CNN was employed to identify crane locations in real-time for the avoidance of safety hazards to their surroundings on construction sites.

Some state-of-the-art models have been developed such as Faster R-CNN, which have good performance in vision-based safety detection under various visual conditions of the construction site. However, there are still some challenges that need to be resolved. Firstly, large datasets are needed to train deep learning models, since existing public datasets of unsafe behaviors in construction are not as large as meeting the requirement of training. Secondly, similarly to vision-based 3D reconstruction, occlusions are also the major challenge that affects the performance of proposed detection models. If workers are partially occluded, the precision of detection reduces significantly. One possible solution is to install multiple cameras on the construction sites to achieve full site coverage. Thirdly, there is no standard to evaluate the performance of detection. As different studies use different training datasets with various samples or criteria, it is difficult to compare the performance of different methods. The lack of standard may lead to discrepancies among the evaluation criteria of unsafe behaviors or conditions. 
Therefore, a widely accepted standard shall be developed for the evaluation of construction unsafety detection. Moreover, a deeper or detailed detection is required (e.g. whether to wear PPEs correctly) since most methods just achieve the basic detection (e.g. whether to wear PPEs or not). In addition, although current methods achieve real-time safety management, it may be not enough to prevent accidents. There is a need for the models that can predict these unsafe conditions in the early time and the alarming mechanism that reflect the predictions to related personnel effectively.

\subsubsection{Quality Management}

AI-based image detection technology is proposed to replace manual detection. According to the different objects, defect detection can be classified as sewer detection (Cheng and Wang 2018), tunnel detection (Makantasis et al. 2015), road detection (Zhang et al. 2017), building detection (Perez et al. 2019), etc.

CNN is one of the networks which has been used in detection. Perez et al. (2019) used CNN for building detection, which could identify mould, ruff, and stain. A new algorithm CrackNet, which uses $\mathrm{CNN}$ without pooling layers, was proposed to detect asphalt pavement cracks (Zhang et al. 2017). Compared with the traditional SVM algorithm, the accuracy and F-measure of this algorithm are better. A tunnel detection technique used CNN to extract defect characteristics and classify them by MLP (Makantasis et al. 2015). The detection rate of this method reached 89\%, better than ANN, SVM, KNN, and Ctree.

Faster R-CNN is another popular algorithm, which solves the generation of region proposals (Ren et al. 2015). Cheng and Wang (2018) used it to detect four types of defects in sewer. Cha et al. (2018) used Faster R-CNN to identify concrete and steel. Compared with the traditional CNN method, this method has a better performance in locating defects. To sum up, deep learning has shown good performance in defect detection.

AI-based defect detection is facing the challenge of lacking a large database for training. Meanwhile, a single quality recognition model can only detect limited types of quality defects. There is no generic model that can detect all types of defects simultaneously. What's more, prior technologies are image-based which means they cannot be applied to recognize defects that are not visible.

\subsubsection{Contract Management}

Contract management is defined as the process that manages the creation and execution of contracts efficiently (Chen et al. 2019b), which is complex since it involves various stakeholders (e.g. contractor, client, engineers, government, etc.). Smart contract, a computer protocol that aims to enforce contracts in a digital way, makes automated contract execution possible (Di Giuda et al. 2020). For example, a term of contract can be written in code and automatically executed by linking related records from government agencies (e.g. government regulations) to blockchain standards (Mason 2017). In addition, once the defined obligations have been completed, the payment function of smart contracts will be executed automatically to pay related contractors for the completed work (Di Giuda et al. 2020). Even further, AI can be used to develop an engine that helps people make decisions based on the information received. For example, the AI engine can decide whether the project continues under different weather conditions based on information from meteorological department and related contract terms. However, there are some limitations of the application of smart contracts, e.g. the inherent security 
vulnerabilities of the input of evidence of fulfilment, machine-readable script of contract that should be able to be reviewed and verified by experts (Nawari and Ravindran 2019a).

\section{CONCLUSIONS}

This paper reviews techniques that contribute to the development of next generation AIbased platform and the revolution of construction management field. Nowadays, the application of AI technology to various practices in the AEC industry brings convenience and improve efficiency for the management work during the real-life construction projects. With the integration of AI, big data, BIM, AR/VR, and blockchain, the platform has great potentials to lead a new revolution in the AEC industry. The automated generation of 3D as-planned and as-built models significantly reduces the time and costs required for drawings and model production, and thus decreases the risks of delay and cost overruns. With the integration of blockchain technology, the AI-based platform can achieve an immutable and traceable documentation mechanism, which resolves the problems of lack of trust in traditional construction projects. The application of AR technology helps develop immersive interaction between real projects and stakeholders (e.g. clients, contractors, engineers), which makes online management more effective. In construction management field, AI brings a new direction for achieving convenient and efficient management of progress, safety, and quality in construction projects, especially for those that are on the remote sites. Under the support of blockchain and AI, automated contract execution can be achieved, including automated payment, real-time updates of referred government regulations, contract execution under unexpected weather, and etc. As more and more studies focus on this field, AI technology shows its vitality and broad prospects in the next generation collaboration.

However, existing platforms are still in its early stage and more studies are needed to make each required technique meet the requirements in the real-life construction projects. The large dataset for training is a common problem for the deep learning application in construction projects. In addition, the expensive computing costs and the low ability of generalization of the deep learning models requires future work to improve. The current AI-based As-planned BIM model generation methods cannot integrate schedule, which is the key to the progress monitoring. For as-built BIM model generation, there is a need for an end-to-end algorithm to efficiently achieve the production. Besides, As-built BIM model generation and progress management are both facing the challenge of occlusions and noise. The equipment and workers may block the construction elements behind them and they often move instead of being still. This situation reduces the accuracy of the system. Similar problems also occur in safety and quality management. Blockchain-based collaboration is still a framework, thus more work needs to be done. Localization is still a problem for AR, and it is difficult for current mobile devices to run AI algorithms to locate. For safety management and quality management, lacking effective datasets that have diverse data limits the performance, and it is hard to create a uniform dataset that suits all conditions. Besides, a widely accepted standard is required for the performance evaluation of detection. An effective alarming mechanism shall be established to reflect the detected results to related personnel. Finally, for AI in the AEC industry, the fragmentation of knowledge remains unresolved. A platform for integrating multiple technologies is still needed to achieve the "end-to-end" construction management. 


\section{REFERENCES}

Aggarwal, S., \& Kumar, N. (2019). Path planning techniques for unmanned aerial vehicles: A review, solutions, and challenges. Computer Communications.

Aoki, Y., Goforth, H., Srivatsan, R. A., \& Lucey, S. (2019). Pointnetlk: Robust \& efficient point cloud registration using pointnet. Proceedings of the IEEE Computer Society Conference on Computer Vision and Pattern Recognition. https://doi.org/10.1109/CVPR.2019.00733

Asadi, K., Ramshankar, H., Noghabaei, M., \& Han, K. (2019). Real-time image localization and registration with BIM using perspective alignment for indoor monitoring of construction. Journal of Computing in Civil Engineering. https://doi.org/10.1061/(ASCE)CP.1943-5487.0000847

Baek, F., Ha, I., \& Kim, H. (2019). Augmented reality system for facility management using image-based indoor localization. Automation in Construction, 99, 18-26.

Bassier, M., Van Genechten, B., \& Vergauwen, M. (2019). Classification of sensor independent point cloud data of building objects using random forests. Journal of Building Engineering, 21, 468-477.

Belgiu, M., \& Drăguţ, L. (2016). Random forest in remote sensing: A review of applications and future directions. ISPRS Journal of Photogrammetry and Remote Sensing, 114, 24-31.

Besl, P. J., \& McKay, N. D. (1992). A Method for Registration of 3-D Shapes. IEEE Transactions on Pattern Analysis and Machine Intelligence. https://doi.org/10.1109/34.121791

Braun, A., \& Borrmann, A. (2019). Combining inverse photogrammetry and BIM for automated labeling of construction site images for machine learning. Automation in Construction, 106, 102879.

Breiman, L. (2001). Random forests. Machine Learning, 45(1), 5-32.

Cha, Y., Choi, W., Suh, G., Mahmoudkhani, S., \& Büyüköztürk, O. (2018). Autonomous structural visual inspection using region-based deep learning for detecting multiple damage types. Computer-Aided Civil and Infrastructure Engineering, 33(9), 731-747.

Chang, W.-C., \& Pham, V.-T. (2019). 3-D Point Cloud Registration Using Convolutional Neural Networks. Applied Sciences, 9(16), 3273.

Che, E., Jung, J., \& Olsen, M. J. (2019). Object recognition, segmentation, and classification of mobile laser scanning point clouds: A state of the art review. Sensors, 19(4), 810.

Chen, J., Kira, Z., \& Cho, Y. K. (2019a). Deep learning approach to point cloud scene understanding for automated scan to $3 \mathrm{D}$ reconstruction. Journal of Computing in Civil Engineering, 33(4), 04019027.

Chen, Y., Wang, M., \& Li, L. (2019b). A Framework for the Contract Management System in Cloud-Based ERP for SMEs in the Construction Industry. In ICCREM 2019: Innovative Construction Project Management and Construction Industrialization (pp. 1-11). American Society of Civil Engineers Reston, VA.

Cheng, J. C. P., \& Wang, M. (2018). Automated detection of sewer pipe defects in closedcircuit television images using deep learning techniques. Automation in Construction, 95, 155-171.

Cho, C. Y., \& Liu, X. (2017). An automated reconstruction approach of mechanical systems in building information modeling (BIM) using 2D drawings. In Computing in Civil Engineering 2017 (pp. 236-244). 
Czerniawski, T., \& Leite, F. (2019). Semantic Segmentation of Building Point Clouds Using Deep Learning: A Method for Creating Training Data Using BIM to Point Cloud Label Transfer. In Computing in Civil Engineering 2019: Visualization, Information Modeling, and Simulation (pp. 410-416). American Society of Civil Engineers Reston, VA.

Dalal, N., \& Triggs, B. (2005). Histograms of oriented gradients for human detection. 2005 IEEE Computer Society Conference on Computer Vision and Pattern Recognition (CVPR'05), 1, 886-893.

Delgado, J. M. D., Butler, L., Brilakis, I., Elshafie, M., \& Middleton, C. (2018). Structural performance monitoring using a dynamic data-driven BIM environment.

Dharmasiri, T., Spek, A., \& Drummond, T. (2018). Eng: End-to-end neural geometry for robust depth and pose estimation using cnns. Asian Conference on Computer Vision, 625-642.

Di Giuda, G. M., Pattini, G., Seghezzi, E., Schievano, M., \& Paleari, F. (2020). The Construction Contract Execution Through the Integration of Blockchain Technology. In Digital Transformation of the Design, Construction and Management Processes of the Built Environment (pp. 27-36). Springer.

Ding, L., Fang, W., Luo, H., Love, P. E., Zhong, B., \& Ouyang, X. (2018). A deep hybrid learning model to detect unsafe behavior: Integrating convolution neural networks and long short-term memory. Automation in Construction, 86, 118-124.

Fang, Q., Li, H., Luo, X., Ding, L., Luo, H., Rose, T. M., \& An, W. (2018). Detecting nonhardhat-use by a deep learning method from far-field surveillance videos. Automation in Construction, 85, 1-9.

Fang, W., Love, P. E., Luo, H., \& Ding, L. (2020). Computer vision for behaviour-based safety in construction: A review and future directions. Advanced Engineering Informatics, 43, 100980.

Fang, W., Zhong, B., Zhao, N., Love, P. E., Luo, H., Xue, J., \& Xu, S. (2019). A deep learning-based approach for mitigating falls from height with computer vision: Convolutional neural network. Advanced Engineering Informatics, 39, 170-177.

Gao, X., Pishdad-Bozorgi, P., Shelden, D., \& Hu, Y. (2019). Machine learning applications in facility life-cycle cost analysis: A review. In Computing in Civil Engineering 2019: Smart Cities, Sustainability, and Resilience (pp. 267-274). American Society of Civil Engineers Reston, VA.

Golparvar-Fard, M., Savarese, S., \& Peña-Mora, F. (2010). Automated model-based recognition of progress using daily construction photographs and IFC-based 4D models. Construction Research Congress 2010: Innovation for Reshaping Construction Practice, 51-60.

Gondia, A., Siam, A., El-Dakhakhni, W., \& Nassar, A. H. (2020). Machine Learning Algorithms for Construction Projects Delay Risk Prediction. Journal of Construction Engineering and Management, 146(1), 04019085.

Ha, I., Kim, H., Park, S., \& Kim, H. (2018). Image retrieval using BIM and features from pretrained VGG network for indoor localization. Building and Environment, 140, $23-$ 31.

Ham, Y., Han, K. K., Lin, J. J., \& Golparvar-Fard, M. (2016). Visual monitoring of civil infrastructure systems via camera-equipped Unmanned Aerial Vehicles (UAVs): a review of related works. Visualization in Engineering, 4(1), 1.

Hamledari, H., Davari, S., Azar, E. R., McCabe, B., Flager, F., \& Fischer, M. (2017). UAVenabled site-to-BIM automation: Aerial robotic-and computer vision-based 
development of as-built/as-is BIMs and quality control. Construction Research Congress, 336-346.

Hamledari, H., Davari, S., Sajedi, S. O., Zangeneh, P., McCabe, B., \& Fischer, M. (2018). UAV Mission Planning Using Swarm Intelligence and 4D BIMs in Support of VisionBased Construction Progress Monitoring and As-Built Modeling. Construction Research Congress 2018, 43-53.

Han, K. K., \& Golparvar-Fard, M. (2014). Automated Monitoring of Operation-level Construction Progress Using 4D BIM and Daily Site Photologs \textbar Construction Research Congress 2014. (n.d.). Retrieved February 23, 2020, from https://ascelibrary.org/doi/abs/10.1061/9780784413517.106.

Han, K. K., \& Golparvar-Fard, M. (2015). Appearance-based material classification for monitoring of operation-level construction progress using 4D BIM and site photologs. Automation in Construction, 53, 44-57. https://doi.org/10.1016/j.autcon.2015.02.007

Han, K., Degol, J., \& Golparvar-Fard, M. (2018). Geometry- and Appearance-Based Reasoning of Construction Progress Monitoring. Journal of Construction Engineering and Management. https://doi.org/10.1061/(ASCE)CO.1943-7862.0001428.

Hargaden, V., Papakostas, N., Newell, A., Khavia, A., \& Scanlon, A. (2019). The Role of Blockchain Technologies in Construction Engineering Project Management. 2019 IEEE International Conference on Engineering, Technology and Innovation (ICE/ITMC), 1-6.

He, K., Zhang, X., Ren, S., \& Sun, J. (2016). Deep residual learning for image recognition. Proceedings of the IEEE Conference on Computer Vision and Pattern Recognition, 770-778.

Hichri, N., Stefani, C., De Luca, L., Veron, P., \& Hamon, G. (2013). From point cloud to BIM: a survey of existing approaches.

Hochreiter, S., \& Schmidhuber, J. (1997). Long short-term memory. Neural Computation, 9(8), 1735-1780.

Huang, P. H., Matzen, K., Kopf, J., Ahuja, N., \& Huang, J. Bin. (2018). DeepMVS: Learning Multi-view Stereopsis. Proceedings of the IEEE Computer Society Conference on Computer Vision and Pattern Recognition. https://doi.org/10.1109/CVPR.2018.00298

Ibrahim, A., \& Golparvar-Fard, M. (2019). 4D BIM Based Optimal Flight Planning for Construction Monitoring Applications Using Camera-Equipped UAVs. In Computing in Civil Engineering 2019: Data, Sensing, and Analytics (pp. 217-224). American Society of Civil Engineers Reston, VA.

Lamsal, B., \& Kunichika, K. (2019). Development of an AR System for the Advancement of the Tasks in the Construction sites.

Lawin, F. J., Danelljan, M., Tosteberg, P., Bhat, G., Khan, F. S., \& Felsberg, M. (2017). Deep projective 3D semantic segmentation. International Conference on Computer Analysis of Images and Patterns, 95-107.

Lee, J.-Y., Kwon, O.-S., Choi, J.-S., \& Park, C.-S. (2012). A study on construction defect management using augmented reality technology. 2012 International Conference on Information Science and Applications, 1-6.

Lei, L., Zhou, Y., Luo, H., \& Love, P. E. (2019). A CNN-based 3D patch registration approach for integrating sequential models in support of progress monitoring. Advanced Engineering Informatics, 41, 100923.

Li, H., Li, X., Luo, X., \& Siebert, J. (2017). Investigation of the causality patterns of nonhelmet use behavior of construction workers. Automation in Construction, 80, 95103. 
Li, J., Greenwood, D., \& Kassem, M. (2019a). Blockchain in the built environment and construction industry: A systematic review, conceptual models and practical use cases. Automation in Construction, 102, 288-307.

Li, J., Yang, B., Chen, D., Wang, N., Zhang, G., \& Bao, H. (2019b). Survey and evaluation of monocular visual-inertial SLAM algorithms for augmented reality. Virtual Reality \& Intelligent Hardware, 1(4), 386-410.

Lim, J., Janssen, P., \& Stouffs, R. (2018). Automated Generation of BIM Models from 2D CAD Drawings.

Liu, C., Wu, J., Kohli, P., \& Furukawa, Y. (2017). Raster-to-vector: Revisiting floorplan transformation. Proceedings of the IEEE International Conference on Computer Vision, 2195-2203.

Lucas, B. D., \& Kanade, T. (1981). ITERATIVE IMAGE REGISTRATION TECHNIQUE WITH AN APPLICATION TO STEREO VISION.

Macher, H., Landes, T., \& Grussenmeyer, P. (2017). From point clouds to building information models: 3D semi-automatic reconstruction of indoors of existing buildings. Applied Sciences, 7(10), 1030.

Makantasis, K., Protopapadakis, E., Doulamis, A., Doulamis, N., \& Loupos, C. (2015). Deep convolutional neural networks for efficient vision based tunnel inspection. 2015 IEEE International Conference on Intelligent Computer Communication and Processing (ICCP), 335-342.

Mason, J. (2017). Intelligent contracts and the construction industry. Journal of Legal Affairs and Dispute Resolution in Engineering and Construction, 9(3), 04517012.

Maturana, D., \& Scherer, S. (2015). Voxnet: A 3d convolutional neural network for realtime object recognition. 2015 IEEE/RSJ International Conference on Intelligent Robots and Systems (IROS), 922-928.

Minsky, M. (1961). Steps toward artificial intelligence. Proceedings of the IRE, 49(1), 830.

Mitha, A. P., Almekhlafi, M. A., Janjua, M. J. J., Albuquerque, F. C., \& McDougall, C. G. (2013). Simulation and augmented reality in endovascular neurosurgery: lessons from aviation. Neurosurgery, 72(suppl_1), A107-A114.

Mohasses, M. (2019). How AI-chatbots can make Dubai smarter? 2019 Amity International Conference on Artificial Intelligence (AICAI), 439-446.

Nakamoto, S. (2008). Bitcoin: A peer-to-peer electronic cash system," http://bitcoin. org/bitcoin. pdf.

Nath, N. D., Behzadan, A. H., \& Paal, S. G. (2020). Deep learning for site safety: Realtime detection of personal protective equipment. Automation in Construction, 112, 103085.

Navon, R., \& Sacks, R. (2007). Assessing research issues in automated project performance control (APPC). Automation in Construction, 16(4), 474-484.

Nawari, N. O., \& Ravindran, S. (2019a). Blockchain and building information modeling (bim): Review and applications in post-disaster recovery. Buildings, 9(6), 149.

Nawari, N. O., \& Ravindran, S. (2019b). Blockchain and the built environment: Potentials and limitations. Journal of Building Engineering, 100832.

Nguyen, T.-H., Oloufa, A. A., \& Nassar, K. (2005). Algorithms for automated deduction of topological information. Automation in Construction, 14(1), 59-70.

Park, C.-S., Lee, D.-Y., Kwon, O.-S., \& Wang, X. (2013). A framework for proactive construction defect management using BIM, augmented reality and ontology-based data collection template. Automation in Construction, 33, 61-71. 
Park, Y., \& Guldmann, J.-M. (2019). Creating 3D city models with building footprints and LIDAR point cloud classification: A machine learning approach. Computers, Environment and Urban Systems, 75, 76-89.

Perez, H., Tah, J. H. M., \& Mosavi, A. (2019). Deep learning for detecting building defects using convolutional neural networks. Sensors, 19(16), 3556.

Perez-Perez, Y., Golparvar-Fard, M., \& El-Rayes, K. (2019). Artificial Neural Network for Semantic Segmentation of Built Environments for Automated Scan2BIM. In Computing in Civil Engineering 2019: Data, Sensing, and Analytics (pp. 97-104). American Society of Civil Engineers Reston, VA.

Pučko, Z., Šuman, N., \& Rebolj, D. (2018). Automated continuous construction progress monitoring using multiple workplace real time 3D scans. Advanced Engineering Informatics, 38, 27-40.

Qi, C. R., Su, H., Mo, K., \& Guibas, L. J. (2017). PointNet: Deep learning on point sets for 3D classification and segmentation. Proceedings - 30th IEEE Conference on Computer Vision and Pattern Recognition, CVPR 2017. https://doi.org/10.1109/CVPR.2017.16

Ratajczak, J., Marcher, C., Schimanski, C. P., Alice, S., Riedl, M., Matt, D. T., \& Budimex, S. A. (2019). BIM-BASED AUGMENTED REALITY TOOL FOR THE MONITORING OF CONSTRUCTION PERFORMANCE AND PROGRESS.

Ren, S., He, K., Girshick, R., \& Sun, J. (2015). Faster r-cnn: Towards real-time object detection with region proposal networks. Advances in Neural Information Processing Systems, 91-99.

Roberts, D., Bretl, T., \& Golparvar-Fard, M. (2017). Detecting and classifying cranes using camera-equipped UAVs for monitoring crane-related safety hazards. In Computing in Civil Engineering 2017 (pp. 442-449).

Schönberger, J. L., Zheng, E., Frahm, J.-M., \& Pollefeys, M. (2016). Pixelwise view selection for unstructured multi-view stereo. European Conference on Computer Vision, 501-518.

Seyis, S. (2019). Pros and cons of using building information modeling in the aec industry. Journal of Construction Engineering and Management, 145(8), 4019046.

Shang, Z., \& Shen, Z. (2017). Real-time 3D reconstruction on construction site using visual SLAM and UAV. ArXiv Preprint ArXiv:1712.07122.

Singh, S., \& Ashuri, B. (2019). Leveraging Blockchain Technology in AEC Industry during Design Development Phase. In Computing in Civil Engineering 2019: Visualization, Information Modeling, and Simulation (pp. 393-401). American Society of Civil Engineers Reston, VA.

Son, H., Kim, C., \& Kwon Cho, Y. (2017). Automated Schedule Updates Using As-Built Data and a 4D Building Information Model. Journal of Management in Engineering. https://doi.org/10.1061/(ASCE)ME.1943-5479.0000528

Sun, J., Lei, K., Cao, L., Zhong, B., Wei, Y., Li, J., \& Yang, Z. (2020). Text visualization for construction document information management. Automation in Construction, 111, 103048.

Tang, Shu, Shelden, D. R., Eastman, C. M., Pishdad-Bozorgi, P., \& Gao, X. (2019). A review of building information modeling (BIM) and the internet of things (IoT) devices integration: Present status and future trends. Automation in Construction, 101, 127-139.

Tezel, A., Papadonikolaki, E., Yitmen, I., \& Hilletofth, P. (2019). Preparing Construction Supply Chains for Blockchain: An Exploratory Analysis. CIB World Building 
Congress 2019 Constructing Smart Cities, Hong Kong SAR, China, 17-21 June 2019., 6.

Tran, H., Kealy, A., \& Diaz-Vilarino, L. (2018). Shape Grammar Approach to 3D Modeling of Indoor Environments Using Point Clouds. Journal of Computing in Civil Engineering, 33(1), 1-14.

Ummenhofer, B., Zhou, H., Uhrig, J., Mayer, N., Ilg, E., Dosovitskiy, A., \& Brox, T. (2017). DeMoN: Depth and motion network for learning monocular stereo. Proceedings 30th IEEE Conference on Computer Vision and Pattern Recognition, CVPR 2017. https://doi.org/10.1109/CVPR.2017.596

Wang, C., Cho, Y. K., \& Kim, C. (2015). Automatic BIM component extraction from point clouds of existing buildings for sustainability applications. Automation in Construction, 56, 1-13.

Wang, Y., \& Solomon, J. M. (2019). Deep closest point: Learning representations for point cloud registration. Proceedings of the IEEE International Conference on Computer Vision, 3523-3532.

Wei, X., Zhang, Y., Li, Z., Fu, Y., \& Xue, X. (2019). DeepSFM: Structure From Motion Via Deep Bundle Adjustment. ArXiv Preprint ArXiv:1912.09697.

World Economic Forum (2016), Shaping the Future of Construction A Breakthrough in Mindset and Technology, retrived from Feb 20th, 2020, http://www3.weforum.org/docs/WEF Shaping the Future of Construction full rep ort _.pdf.

Wu, J., Cai, N., Chen, W., Wang, H., \& Wang, G. (2019). Automatic detection of hardhats worn by construction personnel: A deep learning approach and benchmark dataset. Automation in Construction, 106, 102894.

Yao, Y., Luo, Z., Li, S., Fang, T., \& Quan, L. (2018). MVSNet: Depth inference for unstructured multi-view stereo. Lecture Notes in Computer Science (Including Subseries Lecture Notes in Artificial Intelligence and Lecture Notes in Bioinformatics). https://doi.org/10.1007/978-3-030-01237-3_47

Yao, Y., Luo, Z., Li, S., Shen, T., Fang, T., \& Quan, L. (2019). Recurrent MVSnet for highresolution multi-view stereo depth inference. Proceedings of the IEEE Computer Society Conference on Computer Vision and Pattern Recognition. https://doi.org/10.1109/CVPR.2019.00567

Zeng, Z., Li, X., Yu, Y. K., \& Fu, C.-W. (2019). Deep Floor Plan Recognition Using a Multi-Task Network with Room-Boundary-Guided Attention. Proceedings of the IEEE International Conference on Computer Vision, 9096-9104.

Zhang, A., Wang, K. C. P., Li, B., Yang, E., Dai, X., Peng, Y., Fei, Y., Liu, Y., Li, J. Q., \& Chen, C. (2017). Automated pixel-level pavement crack detection on 3D asphalt surfaces using a deep-learning network. Computer-Aided Civil and Infrastructure Engineering, 32(10), 805-819.

Zhang, M., Cao, T., \& Zhao, X. (2019). Using Smartphones to Detect and Identify Construction Workers' Near-Miss Falls Based on ANN. Journal of Construction Engineering and Management, 145(1), 04018120.

Zhou, H., Ummenhofer, B., \& Brox, T. (2018). Deeptam: Deep tracking and mapping. Proceedings of the European Conference on Computer Vision (ECCV), 822-838.

Zollmann, S., Hoppe, C., Kluckner, S., Poglitsch, C., Bischof, H., \& Reitmayr, G. (2014). Augmented reality for construction site monitoring and documentation. Proceedings of the IEEE, 102(2), 137-154 